\title{
Dyson Orbitals within the fc-CVS-EOM-CCSD Framework: Theory and Appli- cation to X-ray Photoelectron Spectroscopy of Ground and Excited States ${ }^{\dagger}$
}

\author{
Marta L. Vidal, ${ }^{* a}$ Anna I. Krylov ${ }^{\# b}$ and Sonia Coriani ${ }^{\ddagger a}$
}

We report on the implementation of Dyson orbitals within the recently introduced frozen-core (fc) core-valence separated (CVS) equation-of-motion (EOM) coupled-cluster singles and doubles (CCSD) method, which enables efficient and reliable characterization of core-level states. The ionization potential (IP) variant of fc-CVS-EOM-CCSD, in which the EOM target states have one electron less than the reference, gives access to core-ionized states thus enabling modeling of X-ray photoelectron spectra (XPS) and its time-resolved variant (TR-XPS). Dyson orbitals are reduced quantities that can be interpreted as correlated states of the ejected/attached electron; they enter the expressions of various experimentally relevant quantities. In the context of photoelectron spectroscopy, Dyson orbitals can be used to estimate the strengths of photoionization transitions. We illustrate the utility of Dyson orbitals and fc-CVS-EOM-IP-CCSD by calculating XPS of the ground state of adenine and TR-XPS of the excited states of uracil.

\section{Introduction}

$\mathrm{X}$-ray spectroscopy is a powerful element-specific technique for investigating the elementary composition, electronic and geometric structure of matter. The uses of X-ray based techniques are rapidly expanding and the field is undergoing vigorous development. Thanks to last generation synchrotron radiation sources, $\mathrm{X}$-ray free electron lasers (X-FELs) and high-harmonic generation (HHG) lasers, new disciplines such as X-ray femtochemistry, dynamic X-ray Raman spectroscopy, and femtoscale diffraction scattering have emerged; their applications to essential problems in materials and life sciences are gaining momentum. ${ }^{1-3}$ Pumpprobe techniques exploiting, for example, a UV-pump to promote the system to a valence electronically excited/ionized state, and an X-ray probe to excite or ionize a core electron, enable probing local electronic and structural dynamics of matter on the femtosecond time-scale. ${ }^{1,4}$

\footnotetext{
a DTU Chemistry - Department of Chemistry, Technical University of Denmark, DK2800, Kongens Lyngby, Denmark.

${ }^{b}$ Department of Chemistry, University of Southern California, Los Angeles, California 90089, United States.

$\dagger$ Electronic Supplementary Information (ESI) available: Programmable expressions for Dyson orbitals, relevant Cartesian geometries. See DOI: 10.1039/cXCP00000x/

*E-mail:malop@kemi.dtu.dk

"E-mail:krylov@usc.edu

‡E-mail:soco@kemi.dtu.dk
}

These experimental advances have stimulated the concomitant development of theory and simulation technology to assist in the interpretation of experimental results and in the design of new experiments. Theory can also facilitate screening of suitable candidates for investigation by means of these novel experimental techniques.

Of the many $a b$ initio methods currently available, coupledcluster (CC) and equation-of-motion coupled-cluster (EOM-CC) methods are considered among the most accurate and versatile tools for modeling ground-state properties and various spectroscopies. ${ }^{5-10}$ In recent years, the scope of the applicability of the CC/EOM methods has been extended to inner-shell spectroscopies, ${ }^{4,11-19}$ with significant advances due to the introduction of the core-valence separation (CVS) ${ }^{20}$ as an effective way to target core-level states within conventional solver implementations. Recently, ${ }^{21}$ we reported the implementation of the frozencore (fc) core-valence separation (CVS) EOM-CC method with singles and doubles, fc-CVS-EOM-CCSD. In our illustrative calculations, ${ }^{21,22}$ we focused on the variant of the theory for excitation energies, fc-CVS-EOM-EE-CCSD, and demonstrated its ability to reliably model X-ray absorption spectra (XAS) of molecules in their ground and excited states. Whereas conventional XAS probes unoccupied valence orbitals by core-electron excitation, other core-level spectroscopies afford access to other states. For example, X-ray emission (XES) probes occupied valence states, resonant inelastic X-ray scattering (RIXS) probes both occupied and unoccupied valence states, and X-ray photoelectron spectroscopy (XPS) enables the study of ionized states. The focus of 
this study is on the latter, XPS, and its time-resolved (TR) variant, TR-XPS. The underlying excitation/ionization mechanisms in XAS, XPS, and TR-XPS are illustrated in Figure 1. XAS corresponds to the excitation of a core electron to the unoccupied space, XPS detects the electrons produced by ionization of a core orbital in the ground state, and TR-XPS detects the core-ionized electrons from a precursory valence excited state. XPS is thus an

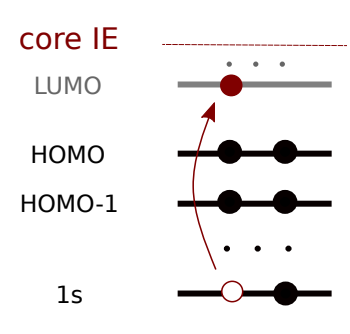

XAS

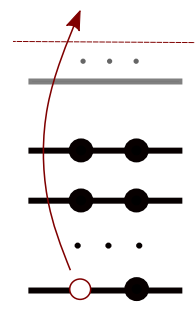

XPS

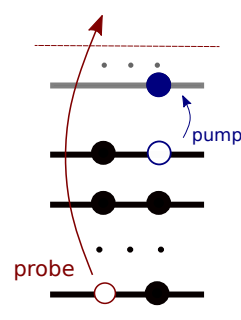

TR-XPS
Fig. 1 Schematic representation of excitation/ionization processes exploited in XAS, XPS and TR-XPS.

extension of UV-VIS photoionization spectroscopy ${ }^{23}$ into the Xray domain and has many attractive features. Since ionization is always an allowed process, photoelectron spectroscopy can probe states that are dark in excitation-based approaches. This is particularly useful for interrogation of dynamics by detecting reaction intermediates that may be optically dark. Even more importantly, charged-particle detection affords high sensitivity, which enables detection of transient species present at low concentrations. Various ingenious experimental setups deliver detailed information about the energy levels and wave-functions from the analysis of the outgoing electron (from its kinetic energy and angular distribution) and of the newly formed ion. In this study, we present the extension of the fc-CVS-EOM-CCSD framework to the calculation of ionization energies and Dyson orbitals for core-ionized states. Dyson orbitals are reduced quantities that can be interpreted as correlated states of the ejected/attached electron; thus, they provide a basis for a rigorous extension of molecular orbital theory to many-body correlated wave functions. ${ }^{24-26}$ Dyson orbitals also enter the expressions of various experimental observables, such as photoionization cross sections; thus, they are necessary for modeling photoelectron spectra. ${ }^{24-30}$ Here we illustrate the utility of Dyson orbitals for computing and interpreting the XPS spectra of ground-state and electronically excited molecules. By using uracil as an example, we illustrate the sensitivity of XPS spectra to the electronic state of the system. We envision that this type of calculations might serve as a guide for the design of future UV-pump/XPS-probe experiments. ${ }^{31}$

\section{Theory}

\section{1 fc-CVS-EOM-CCSD}

The equation-of-motion coupled-cluster method $6,7,32,33$ was originally introduced for calculating excited states. In this variant of the theory, the ground state is treated at the CC level; hence, its wave function is given by the exponential ansatz acting on the reference state $\left|\Phi_{0}\right\rangle$, typically taken as the Hartree-Fock Slater determinant:

$$
\left|\Psi_{\mathrm{CC}}\right\rangle=e^{\hat{T}}\left|\Phi_{0}\right\rangle
$$

$\hat{T}$ is the cluster operator, $\hat{T}=\sum_{\mu} t_{\mu} \hat{\tau}_{\mu}$, where $\hat{\tau}_{\mu}$ are the excitation operators and $t_{\mu}$ are the corresponding cluster amplitudes defined by the CC equations:

$$
\left\langle\Phi_{\mu}\left|\bar{H}-E_{\mathrm{CC}}\right| \Phi_{0}\right\rangle=0 ; \quad E_{\mathrm{CC}}=\left\langle\Phi_{0}|\bar{H}| \Phi_{0}\right\rangle .
$$

Here $\left\langle\Phi_{\mu}\right|$ 's denote $\mu$-tuple excited determinants and $\bar{H}=e^{-\hat{T}} \hat{H} e^{\hat{T}}$ is the similarity transformed Hamiltonian. In the fc-CVS-EOMCCSD method, ${ }^{21}$ the CC equations are solved using the frozencore approximation, which corresponds to setting the groundstate amplitudes (and multipliers) with occupied indices referring to a core orbital to zero. The excited states are then accessed by applying an excitation operator $\hat{R}=\Sigma_{\mu} r_{\mu} \hat{\tau}_{\mu}$ to the ground-state wave function:

$$
\left|\Psi_{\mathrm{R}}\right\rangle=\hat{R} e^{\hat{T}}\left|\Phi_{0}\right\rangle
$$

The amplitudes of the target EOM states are found by diagonalizing the similarity transformed Hamiltonian within a particular sector of Fock space, ${ }^{6-8}$ which is determined by the type of the target states sought and by the method. The choice of the sector in Fock space determines the many-electron basis in which the EOM-CC wavefunctions are represented. For example, in EOM-EE-CCSD this basis comprises the reference, singly, and doubly excited determinants. In fc-CVS-EOM-EE-CCSD, ${ }^{21}$ the manyelectron basis includes only the subset of the determinants in which at least one occupied index corresponds to the core orbital.

Because the operator $\bar{H}$ is non-Hermitian, its left and right eigenstates are distinct

$$
\left\langle L\left|=\left\langle\Phi_{0}|\hat{L} ; \quad| R\right\rangle=\hat{R}\right| \Phi_{0}\right\rangle,
$$

one needs to solve two eigenproblems:

$$
\bar{H}\left|R_{\mu}\right\rangle=E_{\mu}\left|R_{\mu}\right\rangle ; \quad\left\langle L_{\mu}\right| \bar{H}=E_{\mu}\left\langle L_{\mu}\right| .
$$

Thus, in contrast to Hermitian theories, EOM eigenvectors $R$ and $L$ are not adjoints of each other. For properties calculations, it is convenient to normalize them such that they form a biorthogonal set: ${ }^{33}$

$$
\left\langle\Phi_{0} L_{\mu} \mid R_{v} \Phi_{0}\right\rangle=\delta_{\mu v}
$$

By using different types of the excitation operator $\hat{R}$, one can access different sectors of Fock space. In this way, EOM-CC can describe other types of target states, for example, ionized states, which are the focus of this work. In the EOM-IP (EOM for ionization potentials) method, ${ }^{34-36}$ the excitation operator changes the number of electrons in the target state relative to the reference state and hence has the following form:

$$
\hat{R}^{\mathrm{IP}}=\sum_{i} r_{i} \hat{a}_{i}+\frac{1}{4} \sum_{i j a} r_{i j}^{a} \hat{a}_{a}^{\dagger} \hat{a}_{j} \hat{a}_{i}+\ldots,
$$

which allows access to the ionized states:

$$
\left|\Psi_{R}^{N-1}\right\rangle=\hat{R}^{\mathrm{IP}} e^{\hat{T}}\left|\Phi_{0}\right\rangle
$$


Here we consider the EOM-CCSD family of methods in which the cluster operator $\hat{T}$ is truncated to single (S) and double (D) excitations, and so are the $\hat{R}$ and $\hat{L}$ operators. To describe coreionized states, the operators $\hat{R}$ and $\hat{L}$ in fc-CVS-EOM-IP-CCSD are restricted such that they act on at least one core orbital, as prescribed by the core-valence separation scheme. ${ }^{15,20,21}$ The working equations for the fc-CVS-EOM-IP-CCSD $\sigma$-vectors can be found in our earlier work. ${ }^{21}$

\subsection{Dyson Orbitals}

Dyson orbitals are defined as the overlap between an initial $N$ electron and final $N \pm 1$-electron states. In first quantization, the Dyson orbitals are:

$$
\phi_{i f}^{\text {Dyson }}\left(x_{1}\right)=\sqrt{N} \int \Psi_{i}^{N}\left(x_{1}, x_{2}, \ldots, x_{N}\right) \Psi_{f}^{N-1}\left(x_{2}, \ldots, x_{N}\right) d x_{2} \ldots d x_{N} .
$$

This definition does not assume any particular ansatz — Dyson orbitals can be obtained for any pair of wavefunctions, ranging from the exact (full configuration interaction) to pseudonon-interacting electrons ansätze. Within the Hartree-Fock and Koopmans approximations, Dyson orbitals are equal to canonical Hartree-Fock orbitals. When computed for many-body wave functions (e.g., within the CC/EOM formalism), Dyson orbitals include the effect of electron correlation. Thus, they afford rigorous extension of molecular orbital theory to correlated manybody states. As per Eq. (9), the norm of a Dyson orbital can be anything between zero (i.e., as for the two states that are not connected by one-electron ionization) and one (as for the two states that differ by exactly one molecular orbital). Within the HartreeFock/Koopmans approximation, the norms of Dyson orbitals are exactly one. Inclusion of correlation leads to smaller values.

Dyson orbitals can be represented as an expansion over the set of molecular orbitals $\left\{\phi_{p}\right\}$ :

$$
\phi_{i f}^{\text {Dyson }}\left(x_{1}\right)=\sum_{p} \gamma_{p} \phi_{p}\left(x_{1}\right) .
$$

In the second quantization formalism, the coefficients of the expansion (also known as Dyson amplitudes) can be written as:

$$
\begin{aligned}
& \gamma_{p}^{R}=\left\langle\Psi^{N}\left|a_{p}^{\dagger}\right| \Psi^{N-1}\right\rangle, \\
& \gamma_{p}^{L}=\left\langle\Psi^{N-1}\left|a_{p}\right| \Psi^{N}\right\rangle,
\end{aligned}
$$

where superscripts $R$ and $L$ denote right and left Dyson orbitals, respectively. In Hermitian theories, left and right Dyson orbitals are simply (complex) conjugates of each other, but within the EOM-CC framework they are different. General expressions for the EOM-CC Dyson orbitals have been reported before. ${ }^{37}$ Here we extend the formalism to the fc-CVS-EOM-CCSD ansatz, which enables description of core-ionized states. The programmable expressions for the fc-CVS-EOM-CCSD Dyson orbitals are given in the ESI. To derive the equations from the general expressions, we followed the same strategy as for the derivation of the amplitude equations, i.e., we split each set of molecular orbital coefficients into three blocks: virtual, occupied valence (denoted with a $v$ subindex), and occupied core (denoted with a capital later), and retained only those terms that do not vanish due to the frozen core or the CVS constraint. We implemented the resulting expressions in the Q-Chem package ${ }^{38,39}$ using the libtensor library ${ }^{40}$ and the fc-CVS-EOM-IP-CCSD wavefunctions. ${ }^{21}$

Dyson orbitals enter the expressions of the photoionization/photodetachment cross sections ${ }^{30,41}$ as photoelectron matrix element, $D_{k}^{i f}$ :

$$
D_{k}^{i f} \equiv \mathbf{u}\left\langle\phi_{i f}^{\text {Dyson }}|\mathbf{r}| \Psi_{k}^{e l}\right\rangle,
$$

where $\mathbf{r}$ is the dipole moment operator, $\mathbf{u}$ is a unit vector in the direction of polarization of light, and $\Psi_{k}^{e l}$ is the wave function of the ejected electron with wave vector $k$. Thus, for quantitative calculations of total and differential cross sections, one needs both Dyson orbitals and $\Psi_{k}^{e l}$. The latter can be approximated by plane or Coulomb waves, which often yields good agreement with the experimental cross sections. ${ }^{25,30}$ Alternatively, the Dyson orbital can be coupled with continuum functions obtained at the density functional theory level with a multicenter basis of B-spline functions. ${ }^{42,43}$ Once Dyson orbitals are obtained, cross sections calculations can be carried out using, for instance, the ezDyson code. ${ }^{41}$ Since the probability of photoionization is proportional to the matrix elements between the raw, not normalized Dyson orbitals, Eq. (9), and the free-electron state, the norm of the Dyson orbital, which quantifies the extent of the Koopmans character of the transition, can be used as a crude bound of the intensity. ${ }^{26}$ That is why the squared norms of Dyson orbitals defined as

$$
\left\|\phi^{\text {Dyson }}\right\|^{2}=\sum_{q} \gamma_{q}^{2}=R_{F}
$$

are often called spectral strength (or pole strength, or spectroscopic factor) of the $\Psi^{N} \rightarrow \Psi^{N-1}$ transition. Although more quantitative calculations are possible, ${ }^{41,42}$ the Dyson norms can be used for a quick estimate of the ionization intensities in simulations of XPS spectra, ${ }^{29}$ in the same fashion as in valence photoionization studies. ${ }^{26}$ Because of the non-hermiticity of the similarity transformed Hamiltonian, the norms of the EOM-CC right and left Dyson orbitals are not uniquely defined. A possible solution, suggested by the full expressions of the cross sections, ${ }^{25,30,41}$ is to take a geometric average

$$
\left\|\phi^{\text {Dyson }}\right\|^{2}=\left\|\phi_{L}^{\text {Dyson }}\right\| \times\left\|\phi_{R}^{\text {Dyson }}\right\|
$$

as EOM-CC pole strengths. This is consistent with the definition of other inter-state properties within the EOM-CC theory. 33

\section{Computational details}

All calculations were carried out with the Q-Chem electronic structure package. ${ }^{38,39}$ The ionized states were characterized by fc-CVS-EOM-IP-CCSD ${ }^{21}$ and the respective Dyson orbitals. The calculations for $9 \mathrm{H}$-adenine were performed at the planar geometry optimized at the B3LYP/cc-pVTZ level taken from Ref. 44. For uracil, we considered several geometries, all planar. For the ground state, the geometry was optimized at the MP2/cc-pVTZ, whereas the structures for the $S_{1}$ and $S_{2}$ excited states correspond to two stationary points obtained at the EOM-CCSD/aug-cc-pVDZ level of theory. 
The choice of a basis set for calculations of core-level states requires some care. The largest effect of the removal of a core electron is the collapse of valence orbitals toward the nucleus: from the perspective of a valence electron, the removal of a $1 s$ electron is roughly equivalent to adding a proton to the nucleus. To describe such collapse, the basis set must have significant radial flexibility; angular flexibility is much less important. The (radial) collapse of the core orbitals is also significant. To derive a basis set with sufficient flexibility to describe strong orbital relaxation effects, we employed Pople's triple-zeta basis (6-311G**) 45,46 augmented by 2 sets of diffuse functions ${ }^{46}$ on heavier atoms (C, N, $\mathrm{O}$ ) and one on light atoms $(\mathrm{H})$, and with the core functions uncontracted, following the strategy used by Gill and co-workers. ${ }^{47}$ All fc-CVS-EOM-CC calculations reported here employed this 6$311(2+,+) \mathrm{G}^{* *}$ basis set with uncontracted core, as detailed below. For adenine, the basis set was uncontracted on carbon or nitrogen atoms according to the edge of interest, to slightly reduce the computational cost. Table S3 and Figure S3 in the ESI compares the results at the $\mathrm{C}$ K-edge in adenine obtained using the uncontracted core on both $\mathrm{C}$ and $\mathrm{N}$, as well as the Dunning basis sets aug-cc-pVTZ and aug-cc-pCVTZ on the relevant edge atoms and aug-cc-pVDZ on the remaining atoms. We note that Pople's set has fewer basis functions compared to the two combinations of Dunning's sets; therefore, it affords a more favorable computational cost. For uracil, the basis set was uncontracted on carbon, nitrogen, and oxygen atoms for all edges.

The (ground-state) XPS experimental data were taken from Ref. 48 for adenine and from Ref. 49 for uracil. All experimental spectra were digitized from the original references using WebPlotDigitizer. ${ }^{50}$ The theoretical X-ray photoelectron spectra were obtained by convolution of the computed ionization energies and Dyson norms, as per Eq. (14), with a Lorentzian function (FWHM $=0.4 \mathrm{eV}$ ). The computed spectra were shifted for the best alignment with the experimental spectra. No experimental data are yet available for the TR-XPS.

The natural transition orbitals (NTOs) and Dyson orbitals were visualized using MOLDEN. ${ }^{51}$

\section{Results and discussion}

\subsection{X-ray Photoelectron Spectra of Adenine}

Figures 2 and 3 show the X-ray photoelectron spectra of adenine computed at the carbon and nitrogen K-edges, respectively.

\subsubsection{Carbon K-edge XPS}

Figure 2 shows the theoretical (top panel) and the experimen$\mathrm{tal}^{48}$ (bottom panel) carbon K-edge XPS spectra of adenine. The computed spectrum has been uniformly shifted by $-0.5 \mathrm{eV}$ to align it with the first experimental peak at $291.0 \mathrm{eV}$. (With reference to the basis set analysis in the ESI (see Table S3 and Figure S3), the two uncontracted Pople sets yield the results nearly identical to each other and to those obtained with the aug-ccpVTZ/aug-pVDZ basis, and with a slightly larger uniform shift compared to those obtained with aug-cc-pCVTZ/aug-pVDZ.)

The experimental spectrum exhibits two main peaks that are well reproduced by the theoretical spectrum. The analysis of Dyson orbitals reveals that the first feature is due to the ioniza-

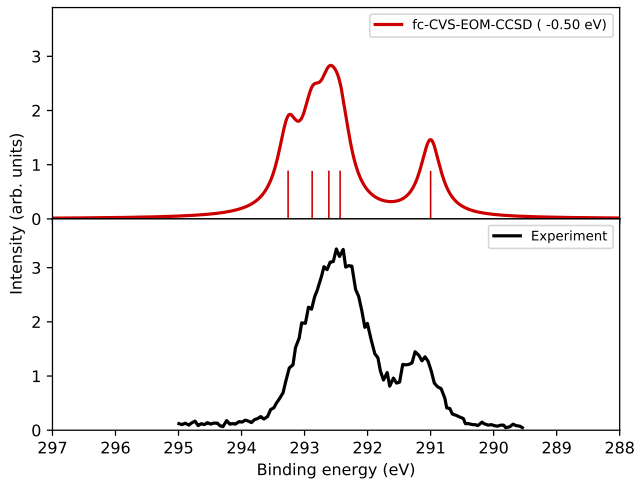

Fig. 2 Adenine. Carbon K-edge fc-CVS-EOM-CCSD/6-311(2+,+) $\mathrm{G}^{* *}$ (uncontracted on C) X-ray photoelectron (XPS) spectra obtained by convolution of the computed ionization energies and Dyson norms with a Lorentzian function (FWHM $=0.4 \mathrm{eV}$ ). The uniform shift applied is given in parenthesis in the legend. The shift was determined with respect to the first experimental peak position, reported to be at $291.0 \mathrm{eV}$. The experimental spectrum was digitized from Ref. 48.

Table 1 Adenine. Carbon K-edge ionization energies (IE, eV) and Dyson orbitals obtained at the fc-CVS-EOM-CCSD/6-311(2+,+)G** (uncontracted on $\mathrm{C}$ ) level of theory

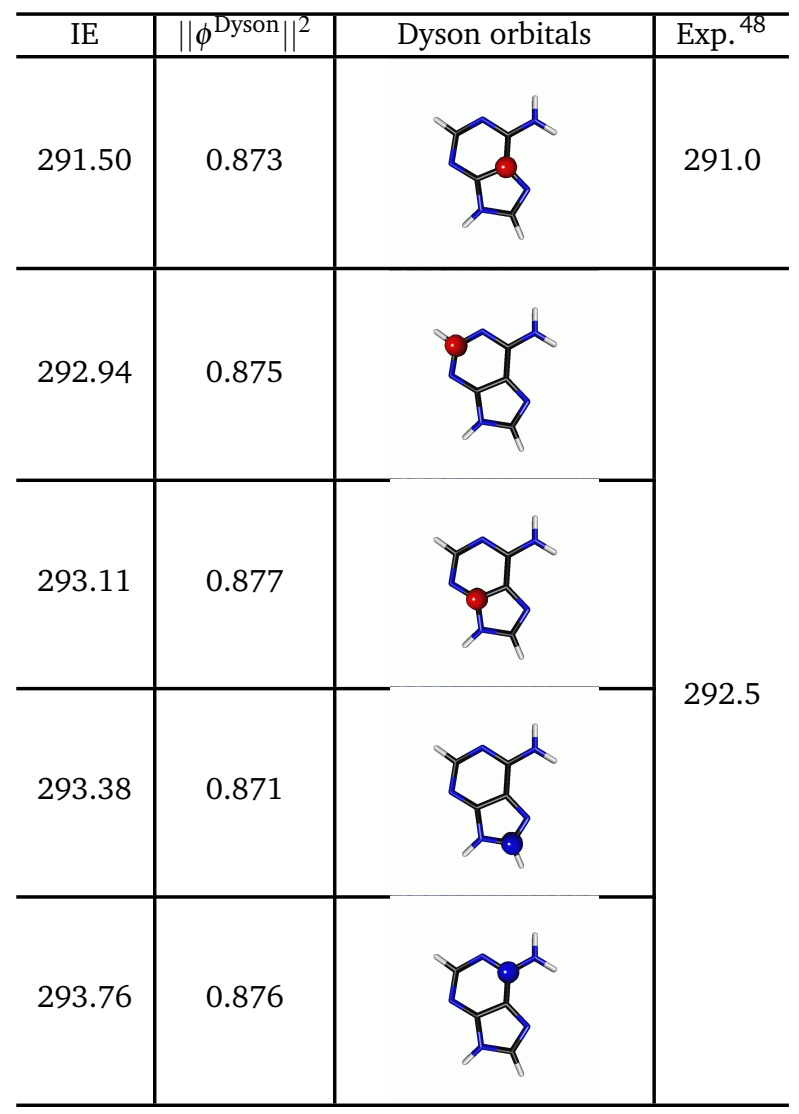

tion of one of the carbon atoms, whereas the second band arises from the ionization of the four other carbon atoms. The Dyson orbitals corresponding to the ionization of the five carbon atoms are displayed in Table 1, along with the ionization energies and pole strengths, and clearly reveal from which atom the $1 s$ elec- 
tron is being ionized at each photon energy. As expected for core-level states, the shapes of the Dyson orbitals are close to the shapes of the canonical Hartree-Fock orbitals and their norms are relatively uniform, ranging between 0.7-0.8. Thus, in this example, the main impact of electron correlation is on the ionization energies. To illustrate the impact of electron correlation, Figure S2 in the ESI compares the C K-edge XPS spectra computed with fc-EOM-IP-CCSD and within Hartree-Fock/Koopmans approximation. We note, in particular, the large difference in the uniform shift required to align the computed spectra to match the experiment: $-0.5 \mathrm{eV}$ for fc-CVS-EOM-CCSD versus $-15.32 \mathrm{eV}$ for Hartree-Fock/Koopmans. Our assignments of the peaks in XPS agrees with the assignment given in Ref. 48 on the basis of ADC calculations. Plekan and coworkers ${ }^{48}$ also reported a theoretical spectrum computed at the ADC(4)/6-31G level of theory. The two methods are in good agreement with each other, however, the overall shift is smaller in the present study. A slight difference, arising from the spanning of the peaks, is the appearance of a shoulder on the high-energy part of the second band, whereas the ADC spectrum shows a shoulder in the low-energy part, and none can be seen in the experiment.

\subsubsection{Nitrogen K-edge XPS}

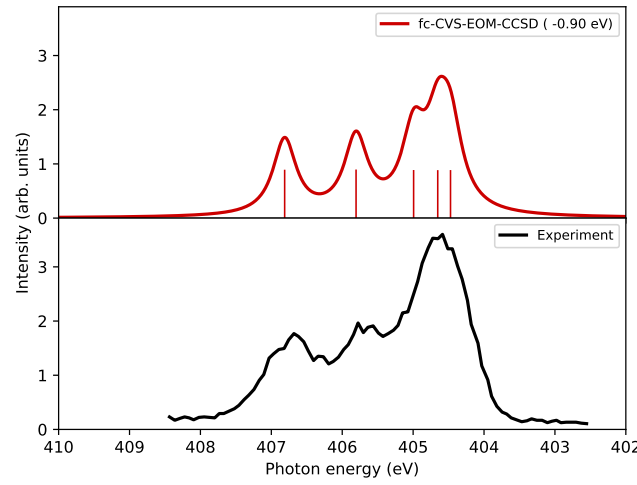

Fig. 3 Adenine. Nitrogen K-edge fc-CVS-EOM-CCSD/6-311(2+,+)G** (uncontracted on N) X-ray photoelectron spectra obtained by convolution of the computed ionization energies and Dyson norms with a Lorentzian function $(F W H M=0.4 \mathrm{eV}$ ). The rigid shift applied is indicated in parenthesis in the legend. It was determined with respect to the first experimental peak position, estimated to be at $404.4 \mathrm{eV}$. The experimental spectrum was digitized from Ref. 48.

The simulated nitrogen K-edge XPS spectrum of adenine, shown in Figure 3, agrees well with the experimental one, after aligning the two first peaks with a shift of $-0.9 \mathrm{eV}$. Although the absolute value of the shift is twice as large as the shift for the carbon K-edge spectrum, the relative value is about the same, i.e., roughly $\sim 0.2 \%$ of the respective ionization energy. One can identify three main bands; the first one originates from the ionization of three different nitrogen atoms resulting in the most intense one. The two other $\mathrm{N}$ atoms, being farther in energy, give rise to two distinct peaks. The Dyson orbitals and the tabulated data for these ionizations are given in Table 2. Hence, the first band is due to the ionization of the three $1 s$ orbitals of the nitro-
Table 2 Adenine. Nitrogen K-edge ionization energies (IE, eV) and Dyson orbitals obtained at the fc-CVS-EOM-CCSD/6-311(2+,+) G** (uncontracted on $\mathrm{N}$ ) level of theory

\begin{tabular}{|c|c|c|c|}
\hline $\mathrm{IE}$ & $\left.\left|\phi^{\text {Dyson }}\right|\right|^{2}$ & Dyson orbital & Exp. ${ }^{48}$ \\
\hline 405.37 & 0.872 & & \\
\hline 405.55 & 0.870 & & 404.4 \\
\hline 405.89 & 0.873 & & \\
\hline 406.70 & 0.882 & & 405.7 \\
\hline 407.71 & 0.878 & & 406.7 \\
\hline
\end{tabular}

gen atoms with double bonds, whereas the second and third peak correspond to the amino group and the remaining $\mathrm{N}$ respectively. As in the case of $\mathrm{C} 1 \mathrm{~s}$ ionizations, our assignment of the nitrogen edge spectrum agrees with Ref. 48.

\subsection{TR-XPS: Uracil}

In the previous section we focused on ground-state XPS. However, XPS can, in principle, also be used to study excited states by means of time-resolved experiments. In this section, we illustrate this idea by calculating photoelectron spectra obtained by ionizing core electrons of the two lowest excited states of uracil. The excited-state dynamics in this system has been investigated by several groups with a variety of approaches. ${ }^{52-58}$ We consider two lowest valence excited states, which are involved in photoinduced dynamics: the first $n \pi^{*}$ dark state (labeled $S_{1}$ ) and the first $\pi \pi^{*}$ bright state (labeled $S_{2}$ ); their respective NTOs are shown in Table 3. To compute Dyson orbitals corresponding to ionization of excited states, one needs to compute matrix elements between the initial excited state (which can be described by EOM-EE using ground state closed-shell reference) and the target ionized state. In the case of valence ionization, i.e., removing an electron from $n, \pi$, or $\pi^{*}$, the target ionized states can be described by EOM-IPCCSD from the same closed-shell reference as the excited states. For example, ionization from $\pi$ or $\pi^{*}$ yields ... $(n)^{2}(\pi)^{1}\left(\pi^{*}\right)^{0}$ electronic configuration, which corresponds to one-electron ioniza- 
Table 3 Uracil. fc-EOMEE-CCSD/6-311(2+,+)G** (uncontracted) NTOs of the first two valence excited states. NTO isosurface is 0.05 .

\begin{tabular}{l|lll}
\hline Excitation & Hole & $\sigma_{K}^{2}$ & Particle \\
\hline $\mathrm{S}_{1}\left(\mathrm{n}_{O} \pi^{*}\right)$ & &
\end{tabular}

tion of the closed-shell reference.

However, in the case of core-level ionization, the resulting target states correspond to shake-up excitations, i.e., states that have doubly excited character (2-hole-1-particle) with respect to the closed-shell reference. For example, removing a $1 s$ electron from $\mathrm{S}_{1}$ yields $(1 s)^{1} \ldots(n)^{1}\left(\pi^{*}\right)^{1}$ configuration. Consequently, the description of these states at the EOM-CCSD level is poor because triple excitations, which are essential for proper description of the correlation (and orbital relaxation), are absent in the EOM-CCSD ansatz. Nevertheless, we computed these states with EOM-CCSD, in order to assess its performance. The results (shown in ESI, see Figure S1 and Table S1) confirm that these shake-up states appear too high in energy, are heavily mixed with other configurations, and are, overall, very poorly described at the CCSD level.

To circumvent this problem, we use the same strategy we explored in connection with simulations of time-resolved x-ray absorption (TR-XAS) of pyrazine. ${ }^{22}$ Specifically, we simulate TR-XPS by carrying out fc-CVS-EOM-IP-CCSD calculations from the high-spin reference state that has the same orbital occupation as the respective valence excited state (shown in Figure 4). The resulting target states have correct orbital occupation: $(1 s)^{1} \ldots(\pi)^{2}(n)^{1}\left(\pi^{*}\right)^{1}$ and $(1 s)^{1} \ldots(\pi)^{1}(n)^{2}\left(\pi^{*}\right)^{1}$. Their multiplicities, however, are incorrect: ionization of the core electron from the singlet excited state produces a doublet state, whereas ionization of $\beta$ electron from the $\alpha \alpha$ triplet state produces a quartet (removing $\alpha$ electron yields a spin-contaminated doublet). The energy difference between the valence triplet and singlet states of the same orbital occupation is large, however, since the electronic configuration in the valence shell in the reference triplet and the target quartet states is the same, we expect that this energy difference will cancel out, yielding a reasonable estimate of core ionization energies. Detailed configuration analysis of the relevant electronic states is given in the Appendix: the analysis suggests that the leading character of the Dyson orbitals should be reproduced reasonably well by this procedure. Here we report the results obtained by ionizing either an $\alpha$ or a $\beta$ electron. Because in the case of ionization of the $\beta$ electron, both the reference and the target states are spin complete, we expect a better error cancellation of the triplet part in the valence shell. However, we anticipate only minor differences between $\alpha$ and $\beta$ ionizations.

Figures 5,6 , and 7 show the results at the carbon, nitrogen,

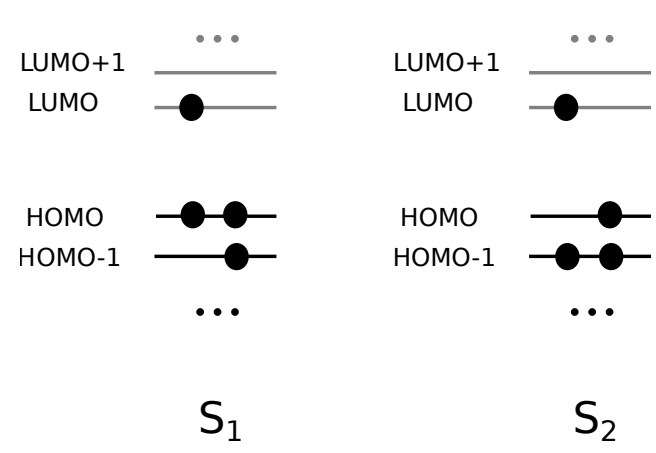

Fig. 4 Schematic representation of the frontier molecular orbitals of the two lowest lying valence excited states of uracil $S_{1}$ and $S_{2}$.

and oxygen K-edges, respectively. In particular, in the upper panels are the spectra for the ground state at its optimized FranckCondon (FC) geometry, in the middle panels are those for the $S_{1}$ states at both the FC (light color) and the $S_{1}$ optimized geometry (dark color) corresponding to the ionization of either the $\alpha$ or $\beta$ electron as indicated on the left-hand side, and similarly for $\mathrm{S}_{2}$ in the lower panels. Each feature in the spectra has its corresponding Dyson orbital assigned on top, these not changing depending on the geometry used. The raw data are summarized in Tables 4, 5 , and 6 for ground, first, and second excited state.

Table 4 Uracil. Ionization energies (IE, eV) and squared norms of Dyson orbitals for the ground state $\left(\mathrm{S}_{0}\right)$.

\begin{tabular}{llll}
\hline K edge & IE & $\left\|\phi^{\text {Dyson }}\right\|^{2}$ & Exp. $^{49}$ \\
\hline Carbon & 291.65 & 0.872 & 291.0 \\
& 293.79 & 0.874 & 292.8 \\
& 295.39 & 0.883 & 294.4 \\
& 296.46 & 0.886 & 295.4 \\
\hline Nitrogen & 407.58 & 0.880 & 406.5 \\
& 408.06 & 0.881 & 406.9 \\
\hline Oxygen & 539.06 & 0.879 & \\
& 539.24 & 0.881 & \\
\hline
\end{tabular}

\subsubsection{Carbon K-edge}

The carbon K-edge XPS spectrum of uracil in the ground state $\left(S_{0}\right)$, shown in the upper panel of Figure 5 , features four peaks of similar intensity. The two higher-energy peaks correspond to the ionization of the carbonyls' $1 s$ carbon orbitals. In the XPS spectra of $S_{2}$, see the two lowest panels of Figure 5, the four peaks are squeezed and blue-shifted relative to $S_{0}$, but each of them still corresponds to the ionization from the same $1 s_{C}$ orbital as in $\mathrm{S}_{0}$. The trend can be rationalized in terms of the stabilization of all core electrons in the excited state due to reduced screening that results in higher energy needed to ionize the core electron (see also Figure 3). Similarly, the first two peaks in the XPS spectra of $S_{1}$ (second and third panel in Figure 5) correspond to the first two in the ground state, apart from the blue shift. However, peak $\mathrm{C}$ is blue-shifted to a larger extent than peak D, becoming the highest in energy. This differential shift results in the two features ( $\mathrm{C}$ and D), originating from the ionization of the two carbonyl groups, coming closer. At the relaxed geometry of $S_{1}$, they practically 
Table 5 Uracil. Ionization energies (IE, eV) and squared norms of Dyson orbitals for the first excited state $\left(\mathrm{S}_{1}\right)$. The results are reported at the Franck-Condon geometry (no label) and at state-specific zero-gradient, stationary point (label $s p$ ) geometry.

\begin{tabular}{|c|c|c|c|c|c|c|c|c|}
\hline \multirow{3}{*}{ K-edge } & \multicolumn{4}{|c|}{$S_{1}$} & \multicolumn{4}{|c|}{$\mathrm{S}_{1(s p)}$} \\
\hline & \multicolumn{2}{|c|}{ IE } & \multicolumn{2}{|c|}{$\left|\phi^{\text {Dyson }}\right|^{2}$} & \multicolumn{2}{|c|}{$\mathrm{IE}$} & \multicolumn{2}{|c|}{$\left\|\phi^{\text {Dyson }}\right\|^{2}$} \\
\hline & $\alpha$ & $\beta$ & $\alpha$ & $\beta$ & $\alpha$ & $\beta$ & $\alpha$ & $\beta$ \\
\hline \multirow[t]{4}{*}{ Carbon } & 293.63 & 293.51 & 0.875 & 0.876 & 293.83 & 293.77 & 0.877 & 0.877 \\
\hline & 295.49 & 295.47 & 0.877 & 0.877 & 295.95 & 295.89 & 0.872 & 0.869 \\
\hline & 298.00 & 297.99 & 0.895 & 0.895 & 298.04 & 298.04 & 0.896 & 0.896 \\
\hline & 298.51 & 298.61 & 0.884 & 0.883 & 298.24 & 298.31 & 0.879 & 0.878 \\
\hline \multirow[t]{2}{*}{$\overline{\text { Nitrogen }}$} & 409.41 & 409.38 & 0.883 & 0.884 & 409.63 & 409.58 & 0.881 & 0.881 \\
\hline & 410.26 & 410.12 & 0.888 & 0.889 & 410.32 & 410.24 & 0.889 & 0.890 \\
\hline \multirow[t]{2}{*}{ Oxygen } & 540.66 & 540.65 & 0.887 & 0.887 & 540.65 & 540.64 & 0.887 & 0.888 \\
\hline & 547.02 & 545.93 & 0.882 & 0.896 & 546.83 & 545.59 & 0.880 & 0.894 \\
\hline
\end{tabular}

merge into a single peak of combined higher intensity relative to the same peak in the $\mathrm{S}_{0}$ spectrum. The NTOs in Table 3 show that the change in electron density in $S_{1}$ is localized near the same carbonyl group; therefore, such a change in the ionization energy is expected.

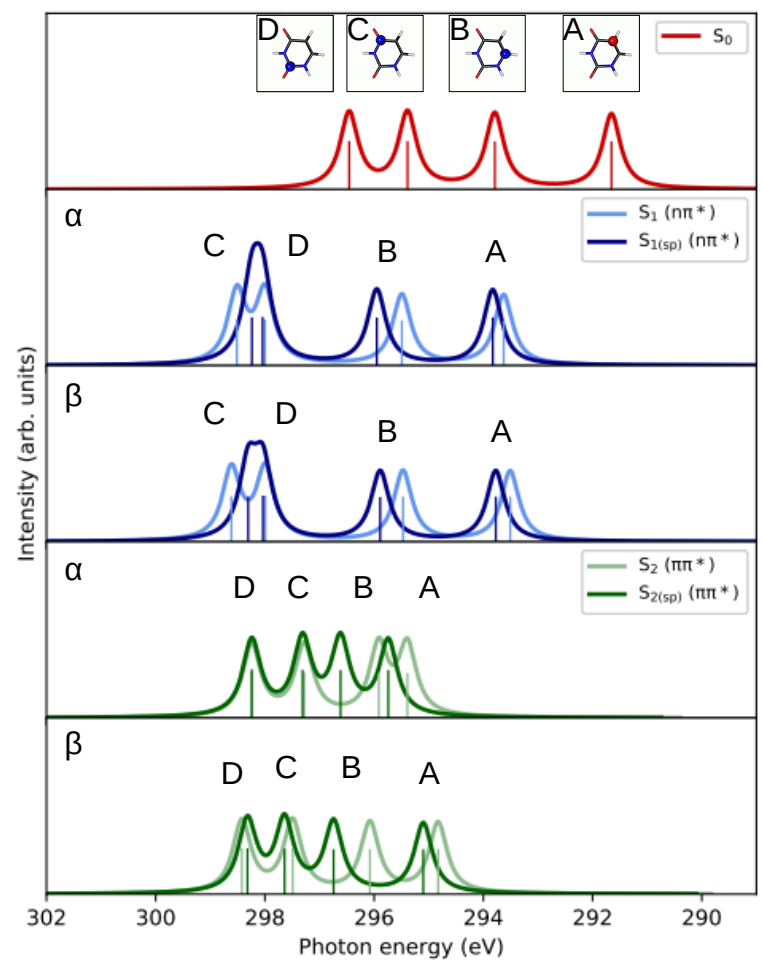

Fig. 5 Uracil. C K-edge fc-CVS-EOM-CCSD/6-311(2+,+) G** (uncontracted) X-ray photoelectron spectra of the ground state, first excited state $\left(S_{1}\right)$ and second excited state $\left(S_{2}\right)$ corresponding to the ejection of either an $\alpha$ or a $\beta$ electron, obtained by convolution of the computed ionization energies and Dyson norms with a Lorentzian function $(\mathrm{FWHM}=0.4 \mathrm{eV})$.

\subsubsection{Nitrogen K-edge}

The XPS spectra and Dyson orbitals of the ground and excited states of uracil at the nitrogen K-edge are shown in Figure 6. At the first glance, the most significant difference in the spectra of the valence excited states relative to the ground state are blue shifts and larger splittings between the peaks. However, the inspection of the Dyson orbitals reveals that peaks A and B are swapped in the $S_{1}$ state with respect to the other two states. This indicates that in the $S_{1}$ excited state there is a larger stabilization of the core electron whose ionization gives rise to peak $A$ than of the electron responsible for peak B. The larger splitting between peaks $A$ and $B$ in the $S_{2}$ spectrum indicates a larger stabilization of the $1 s_{N}$ electron corresponding to peak B relative to the $1 s_{N}$ electron corresponding to peak A.

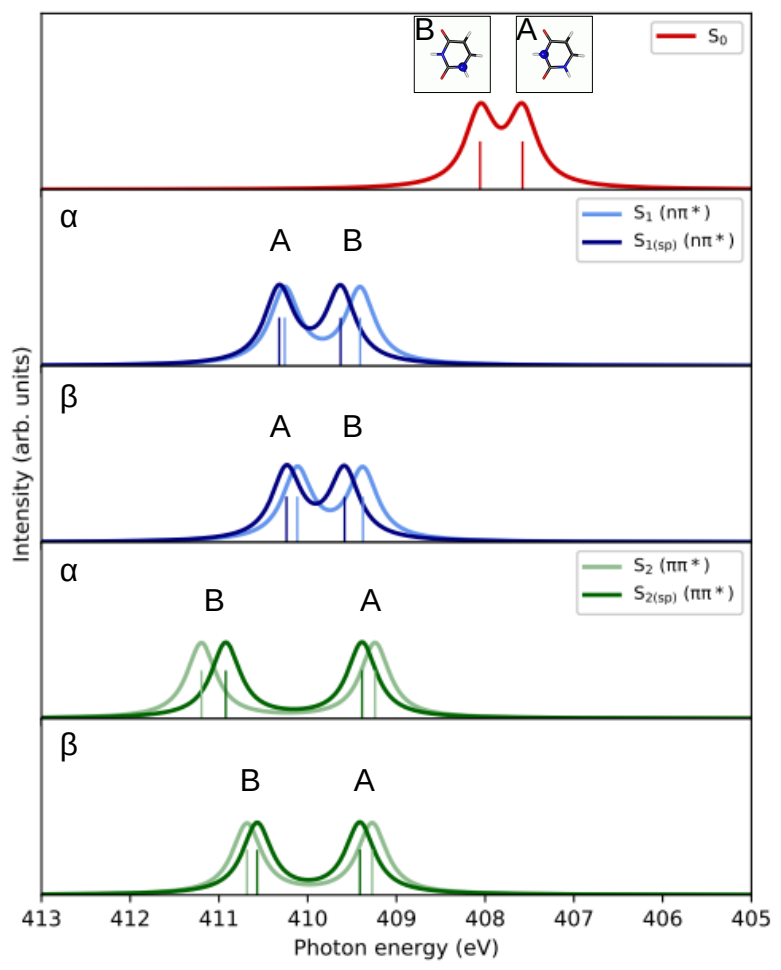

Fig. 6 Uracil. $\mathrm{N}$ K-edge fc-CVS-EOM-CCSD/6-311(2+,+)G** (uncontracted) X-ray photoelectron spectra of the ground state, first $\left(S_{1}, n \pi^{*}\right)$ and second $\left(\mathrm{S}_{2}, \pi \pi^{*}\right)$ excited state corresponding to the ejection of either an $\alpha$ or a $\beta$ electron, obtained by convolution of the computed ionization energies and Dyson norms with a Lorentzian function $(F W H M=0.4 \mathrm{eV})$. 
Table 6 Uracil. Ionization energies (IE, eV) and squared norms of the second excited state $\left(\mathrm{S}_{2}\right)$. The results are reported at the Franck-Condon geometry (no label) and at state-specific zero-gradient, stationary point (label $s p$ ) geometry.

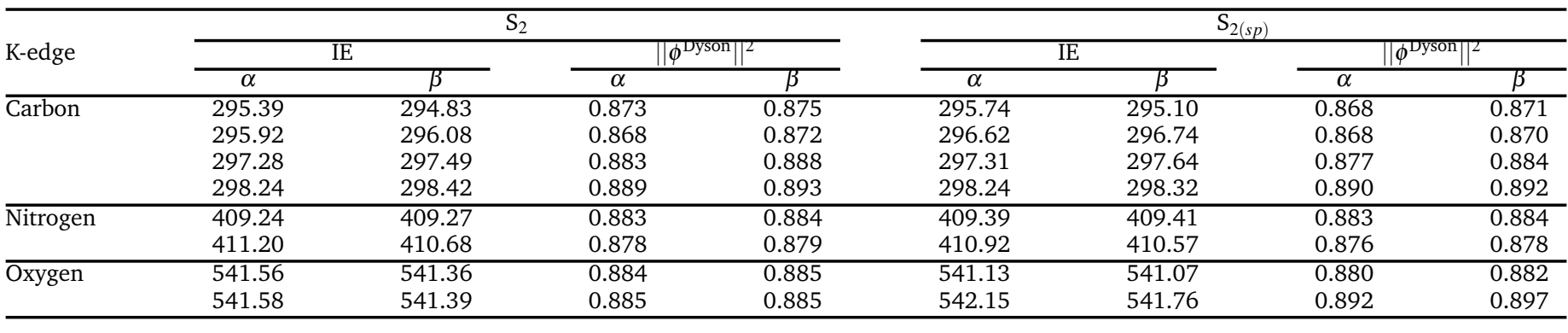

\subsubsection{Oxygen K-edge}

Figure 7 displays the XPS spectra of the ground and excited states at the oxygen K-edge. In this case, there are significant differences between the spectra of the three states. In the ground state there is only one band, originating from the ionization of the two O $1 s$ that are very close in energy, in accordance to their chemical similarities. The spectrum of the $n \pi^{*}$ state shows that the two $\mathrm{O} 1 s$ orbitals are no longer nearly degenerate - their ionization energies now differ by more than $6 \mathrm{eV}$. This is consistent

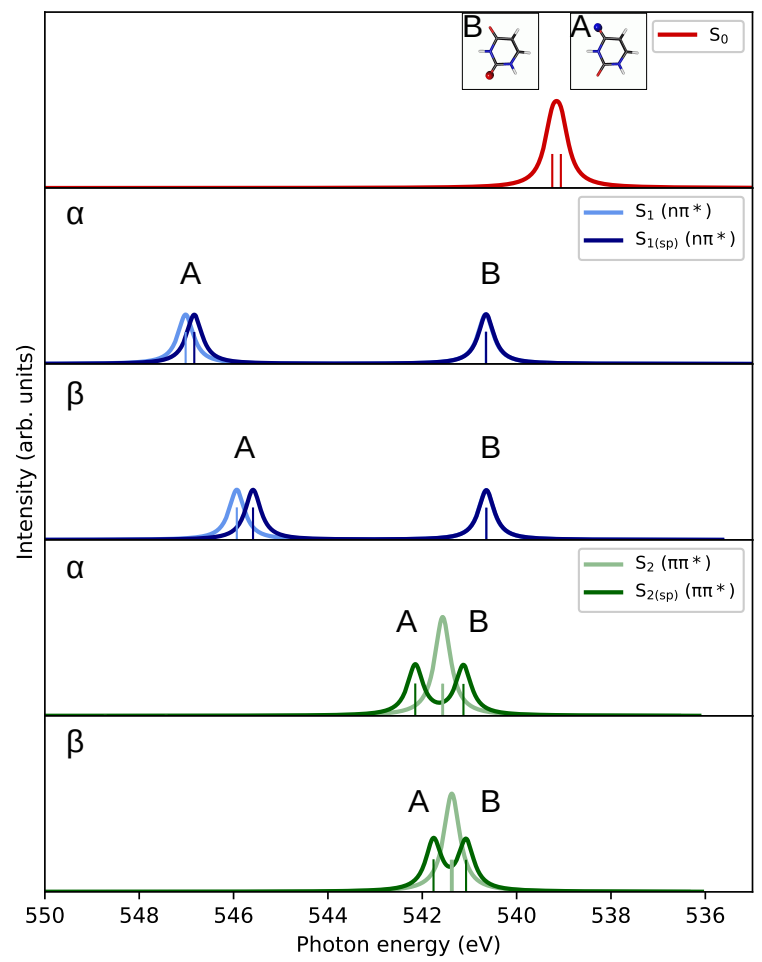

Fig. 7 Uracil. O K-edge fc-CVS-EOM-CCSD/6-311(2+,+)G** (uncontracted) $\mathrm{X}$-ray photoelectron spectra of the ground state, first excited state $\left(S_{1}\right)$, and second excited state $\left(S_{2}\right)$ corresponding to the ejection of either an $\alpha$ or a $\beta$ electron, obtained by convolution of the computed ionization energies and Dyson norms with a Lorentzian function $(F W H M=0.4 \mathrm{eV})$.

with the observation that the $S_{1}$ state is derived by excitation of the electron in the lone pair of oxygen A, so it is quite localized. The reduced screening of this oxygen atom leads to the increased Coulomb attraction of other electrons, including the $1 s$ core electron, resulting in lowering the respective energies (increased IEs). In the $S_{2}$ state, on the other hand, we observe (Table 3 ) changes of electron density on both oxygens, with oxygen A slightly more de-shielded than oxygen $B$, which results in larger stabilization of the core electron corresponding to peak A relative to the electron corresponding to peak B.

\section{Conclusions}

We presented the implementation of the Dyson orbitals within the recently developed fc-CVS-EOM-CCSD framework. The fc-EOMIP-CCSD method enables calculations of core-ionized states, providing a computational tool for modeling XPS and TR-XPS. Dyson orbitals provide a rigorous extension of molecular orbital theory to many-body wave functions; they can be interpreted as correlated states of the ejected electron. Qualitatively, Dyson orbitals can be used for spectral assignments of the features in photoelectron spectra. Quantitatively, they are necessary elements for computing photoelectron cross sections (their norms provide a rough estimate of the intensities). In contrast to valence ionization spectroscopy, the shapes of Dyson orbitals corresponding to $1 s$ coreionized states appear to be very similar to the respective canonical Hartree-Fock orbitals. However, the differences between the Dyson orbitals and canonical Hartree-Fock orbitals may be larger for $L$-edge and below, and also for systems with extensive electronic degeneracies, where correlation can mix the Hartree-Fock orbitals. The reported implementation provides a basis for future work investigating these effects.

To illustrate the possible uses of the developed methodology, we computed the XPS spectra of the ground state of adenine, and of the ground and excited states of uracil. For the latter the calculations reveal significant differences in the computed core-level spectra, suggesting that excited-state dynamics of uracil and similar molecules can be investigated by UV-pump/XPS-probe. As of today, no time-resolved experiments as the ones proposed in this study have been reported. ${ }^{31}$ We hope that our results will stimulate future experimental and theoretical efforts in this direction.

\section{Conflicts of interest}

The authors declare the following competing financial interest(s): A.I.K. is a part owner and a board member of Q-Chem, Inc. 


\section{Acknowledgment}

M.L.V. and S.C. acknowledge support from DTU Chemistry (startup Ph.D. grant). S.C. acknowledges support from the Independent Research Fund Denmark - DFF-RP2 grant no. 7014-00258B and from the H2020-MSCA-ITN-2017 training network "COSINE - COmputational Spectroscopy In Natural sciences and Engineering". A.I.K. acknowledges support by the U.S. National Science Foundation (No. CHE-1566428) and by the Simons Foundation. We thank Prof. Peter Gill from the University of Sydney for his enlightening explanation of core-relaxation effects.

\section{Appendix: Configuration analysis of the ini- tial and target states in excited-state ioniza- tion}

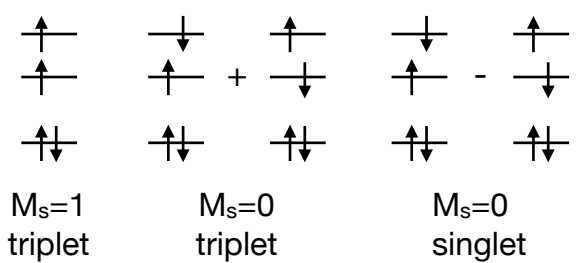

Fig. 8 Spin-adapted wave functions for 4-electrons-in-3-orbitals (only configurations with positive spin projection are shown).

We consider a 4-electrons-in-3-orbitals model to analyze a possible impact of using high-spin triplet reference on Dyson orbitals. Fig. 8 shows electronic configurations of the high-spin triplet state and the $M_{s}=0$ singlet and triplet states. All three wave functions have the same orbital occupation, representing valence excited states. The lowest orbital represents the core orbital, $\phi_{c}$. Ionization of the core orbital can produce open-shell quartet and doublet states. The resulting spin-adapted 3-electrons-in-3orbitals wave functions are shown in Fig. 9 (the complete set of configurations for 3-electrons-in-3-orbitals can be found, for example, in Ref. 59). Ionization of the triplet can produce the quartet and doublets, whereas ionization of the singlet can only result in a doublet. Further analysis of configurations in Fig. 9 makes it evident that one-electron ionization of the triplets can produce (a)-(c), whereas the ionization of the singlet can only yield (d). As expected, the Dyson orbital in all cases equals $\phi_{c}$. Thus, it is reasonable to assume that the leading contributions to the Dyson orbital for ionizing singlet and triplet excited states with the same orbital occupation would be similar.

Let us now analyze whether our computational scheme of calculating Dyson orbitals from a high-spin triplet state suffers from spin contamination. Ionization of the $\beta$-electron from the highspin reference yields high-spin quartet, configuration (a) from Fig. 9; in this calculation both the initial and the target states are spin complete and the Dyson orbital equals $\phi_{c}$. Ionization of $\alpha$-electron yields the third configuration of quartet (b) or the first configuration of doublet (c). It is obvious that the target states would be spin-contaminated, as the two other configurations present in (b) and (c) would be missing. This spin-incompleteness may affect the energies of the target states. However, the effect (a)

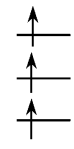

(b)

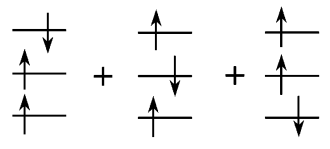

(c)

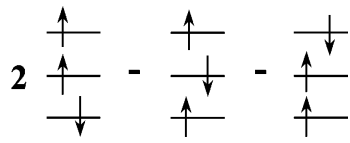

(d)

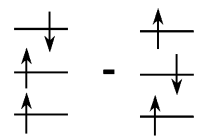

Fig. 9 Spin-adapted wave functions for 3-electrons-in-3-orbitals in which each orbital is singly occupied (only configurations with positive spin projection are shown). Configurations (a) and (b) are quartets; configurations (c) and (d) are doublets. Reproduced with permission from Ref. 59.

is likely to be small because of the small exchange integral between the core and valence orbitals. Most importantly, the spinincompleteness of the target states has no effect on the Dyson orbital, since the missing configurations are connected to the initial state (a) by a three-electron operator involving flipping the spin of the core electron. In the model example with three orbitals, the resulting Dyson orbital equals $\phi_{c}$, just as in the case of properly spin-adapted states. Of course, in systems with more electrons, some spin-contamination will likely be present, however, the leading contributions to the Dyson orbital should be captured by this scheme reasonably well. To estimate the numeric consequences of spin-incompleteness, one can compare the IEs and the square norms of Dyson orbitals computed by ionizing $\alpha$ or $\beta$ electrons from the high-spin $\alpha \alpha$ reference. The results in Tables 5 and 6 (and the corresponding figures) confirm that the differences in IEs are small. The differences in the norms of the Dyson orbitals are in the third digit, which confirms that, as far as Dyson orbitals are concerned, the calculations of ionized states using high-spin triplet reference do not introduce gross errors due to the lack of spin adaptation.

\section{References}

1 P. M. Kraus, M. Zürch, S. K. Cushing, D. M. Neumark and S. R. Leone, Nature Rev. Chem., 2018, 2, 82-94.

2 Z. Li, M. E.-A. Madjet, O. Vendrell and R. Santra, Faraday Discuss., 2014, 171, 475.

3 X-Ray Free Electron Lasers: Applications in Materials, Chemistry and Biology, ed. U. Bergmann, V. Yachandra and J. Yano, Royal Society of Chemistry, 2017.

4 T. Wolf, R. Myhre, J. Cryan, S. Coriani, R. Squibb, A. Battistoni, N. Berrah, C. Bostedt, P. Bucksbaum, G. Coslovich, R. Feifel, K. Gaffney, J. Grilj, T. Martinez, S. Miyabe, S. Moeller, M. Mucke, A. Natan, R. Obaid, T. Osipov, 
O. Plekan, S. Wang, H. Koch and M. Gühr, Nature Commun., 2017, 8, 1-7.

5 O. Christiansen, Theor. Chem. Acc., 2006, 116, 106.

6 A. I. Krylov, Ann. Rev. Phys. Chem., 2008, 59, 433-462.

7 R. J. Bartlett, WIREs Comput Mol Sci, 2012, 2, 126-138.

8 K. Sneskov and O. Christiansen, WIREs Comput. Mol. Sci., 2012, 2, 566-584.

9 T. Helgaker, S. Coriani, P. Jørgensen, K. Kristensen, J. Olsen and K. Ruud, Chem. Rev., 2012, 112, 543-631.

10 J. Wenzel, A. Holzer, M. Wormit and A. Dreuw, J. Chem. Phys., 2015, 142, 214104.

11 S. Coriani, O. Christiansen, T. Fransson and P. Norman, Phys. Rev. A, 2012, 85, 022507.

12 S. Coriani, T. Fransson, O. Christiansen and P. Norman, J. Chem. Theory Comput., 2012, 8, 1616.

13 D. Zuev, E. Vecharynski, C. Yang, N. Orms and A. I. Krylov, J. Comput. Chem., 2015, 36, 273-284.

14 B. Peng, P. J. Lestrange, J. J. Goings, M. Caricato and X. Li, J. Chem. Theory Comput., 2015, 11, 4146.

15 S. Coriani and H. Koch, J. Chem. Phys., 2015, 143, 181103.

16 S. Coriani and H. Koch, J. Chem. Phys., 2016, 145, 149901.

17 A. Sadybekov and A. I. Krylov, J. Chem. Phys., 2017, 147, 014107.

18 R. Faber and S. Coriani, Phys. Chem. Chem. Phys., 2019.

19 K. Nanda, M. L. Vidal, R. Farber, S. Coriani and A. Krylov, Phys. Chem. Chem. Phys., 2019.

20 L. S. Cederbaum, W. Domcke and J. Schirmer, Phys. Rev. A: At. Mol. Opt. Phys., 1980, 22, 206-222.

21 M. L. Vidal, X. Feng, E. Epifanovsky, A. I. Krylov and S. Coriani, J. Chem. Theory Comput., 2019, 15, 3117-3133.

22 S. Tsuru, M. L. Vidal, M. Pápai, A. I. Krylov, K. B. Møller and S. Coriani, J. Chem. Phys.

23 O. Kostko, B. Bandyopadhyay and M. Ahmed, Annu. Rev. Phys. Chem., 2016, 67, 19-40.

24 C. Melania Oana and A. I. Krylov, J. Chem. Phys., 2007, 127, 234106.

25 C. M. Oana and A. I. Krylov, J. Chem. Phys., 2009, 131, 124114.

26 M. Díaz-Tinoco, H. H. Corzo, F. Pawłowski and J. V. Ortiz, Mol. Phys., 2019, 117, 2275-2283.

27 R. Arneberg, J. Müller and R. Manne, Chem. Phys., 1982, 64, $249-258$.

28 J. V. Ortiz, Int. J. Quantum Chem., 2004, 100, 1131-1135.

29 M. Spanner, S. Patchkovskii, C. Zhou, S. Matsika, M. Kotur and T. C. Weinacht, Phys. Rev. A, 2012, 86, 053406.

30 S. Gozem, A. O. Gunina, T. Ichino, D. L. Osborn, J. F. Stanton and A. I. Krylov, J. Phys. Chem. Lett., 2015, 6, 4532-4540.

31 K. Price, 2019, Private communication.

32 H. Sekino and R. J. Bartlett, Int. J. Quant. Chem., 1984, 26, 255-265.

33 J. Stanton and R. Bartlett, J. Chem. Phys., 1993, 98, 70297039.

34 J. Stanton and J. Gauss, J. Chem. Phys., 1994, 101, 8938-
8944.

35 J. Stanton and J. Gauss, Adv. Chem. Phys., 2003, 125, 101146.

36 P. A. Pieniazek, S. E. Bradforth and A. I. Krylov, J. Chem. Phys., 2008, 129, 074104.

37 A. Gunina, Ph.D. thesis, University of Southern California, 2017.

38 A. I. Krylov and P. M. W. Gill, WIREs Comput. Mol. Sci., 2013, 3, 317-326.

39 Y. Shao, Z. Gan, E. Epifanovsky, A. T. Gilbert, M. Wormit, J. Kussmann, A. W. Lange, A. Behn, J. Deng, X. Feng, D. Ghosh, M. Goldey, P. R. Horn, L. D. Jacobson, I. Kaliman, R. Z. Khaliullin, T. Kuś, A. Landau, J. Liu, E. I. Proynov, Y. M. Rhee, R. M. Richard, M. A. Rohrdanz, R. P. Steele, E. J. Sundstrom, H. L. Woodcock III, P. M. Zimmerman, D. Zuev, B. Albrecht, E. Alguire, B. Austin, G. J. O. Beran, Y. A. Bernard, E. Berquist, K. Brandhorst, K. B. Bravaya, S. T. Brown, D. Casanova, C.-M. Chang, Y. Chen, S. H. Chien, K. D. Closser, D. L. Crittenden, M. Diedenhofen, R. A. D. Jr., H. Do, A. D. Dutoi, R. G. Edgar, S. Fatehi, L. Fusti-Molnar, A. Ghysels, A. Golubeva-Zadorozhnaya, J. Gomes, M. W. Hanson-Heine, P. H. Harbach, A. W. Hauser, E. G. Hohenstein, Z. C. Holden, T.-C. Jagau, H. Ji, B. Kaduk, K. Khistyaev, J. Kim, J. Kim, R. A. King, P. Klunzinger, D. Kosenkov, T. Kowalczyk, C. M. Krauter, K. U. Lao, A. D. Laurent, K. V. Lawler, S. V. Levchenko, C. Y. Lin, F. Liu, E. Livshits, R. C. Lochan, A. Luenser, P. Manohar, S. F. Manzer, S.-P. Mao, N. Mardirossian, A. V. Marenich, S. A. Maurer, N. J. Mayhall, E. Neuscamman, C. M. Oana, R. Olivares-Amaya, D. P. O’Neill, J. A. Parkhill, T. M. Perrine, R. Peverati, A. Prociuk, D. R. Rehn, E. Rosta, N. J. Russ, S. M. Sharada, S. Sharma, D. W. Small, A. Sodt, T. Stein, D. Stük, Y.-C. Su, A. J. Thom, T. Tsuchimochi, V. Vanovschi, L. Vogt, O. Vydrov, T. Wang, M. A. Watson, J. Wenzel, A. White, C. F. Williams, J. Yang, S. Yeganeh, S. R. Yost, Z.-Q. You, I. Y. Zhang, X. Zhang, Y. Zhao, B. R. Brooks, G. K. Chan, D. M. Chipman, C. J. Cramer, W. A. Goddard III, M. S. Gordon, W. J. Hehre, A. Klamt, H. F. Schaefer III, M. W. Schmidt, C. D. Sherrill, D. G. Truhlar, A. Warshel, X. Xu, A. Aspuru-Guzik, R. Baer, A. T. Bell, N. A. Besley, J.-D. Chai, A. Dreuw, B. D. Dunietz, T. R. Furlani, S. R. Gwaltney, C.-P. Hsu, Y. Jung, J. Kong, D. S. Lambrecht, W. Liang, C. Ochsenfeld, V. A. Rassolov, L. V. Slipchenko, J. E. Subotnik, T. V. Voorhis, J. M. Herbert, A. I. Krylov, P. M. Gill and M. Head-Gordon, Mol. Phys., 2015, 113, 184-215.

40 E. Epifanovsky, M. Wormit, T. Kuś, A. Landau, D. Zuev, K. Khistyaev, P. U. Manohar, I. Kaliman, A. Dreuw and A. I. Krylov, J. Comput. Chem., 2013, 34, 2293-2309.

41 S. Gozem and A. I. Krylov, 2015, ezDyson, http://iopenshell.usc.edu/downloads/.

42 H. Bachau, E. Cormier, P. Decleva, J. E. Hansen and F. Martín, Rep. Prog. Phys., 1999, 64, 1815.

43 A. Ponzi, C. Angeli, R. Cimiraglia, S. Coriani and P. Decleva, J. Chem. Phys., 2014, 140, 204304.

44 F. Santoro, R. Improta, T. Fahleson, J. Kauczor, P. Norman and S. Coriani, J. Phys. Chem. Lett., 2014, 5, 1806-1811. 
45 R. Krishnan, J. Binkley, R. Seeger and J. Pople, J. Chem. Phys., 1980, 72, 650.

46 T. Clark, J. Chandrasekhar and P. Schleyer, J. Comput. Chem., 1983, 4, 294-301.

47 N. Besley, A. Gilbert and P. Gill, J. Chem. Phys., 2009, 130, 124308.

48 O. Plekan, V. Feyer, R. Richter, M. Coreno, M. de Simone, K. Prince, A. Trofimov, E. Gromov, I. Zaytseva and J. Schirmer, Chem. Phys., 2008, 347, 360-375.

49 V. Feyer, O. Plekan, R. Richter, M. Coreno, M. de Simone, K. C. Prince, A. B. Trofimov, I. L. Zaytseva and J. Schirmer, J. Phys. Chem. A, 2010, 114, 10270-10276.

50 A. Rohatgi, WebPlotDigitizer, version 4.2, https://automeris.io/WebPlotDigitizer, 2019.

51 G. Schaftenaar and J. Noordik, J. Comput.-Aided Mol. Design, 2000, 14, 123-134.
52 S. Matsika, J. Phys. Chem. A, 2004, 108, 7584-7590.

53 F. Santoro, V. Barone, T. Gustavsson and R. Improta, J. Am. Chem. Soc., 2006, 128, 16312-16322.

54 H. Hudock, B. Levine, A. Thompson, H. Satzger, D. Townsend, N. Gador, S. Ulrich, A. Stolow and T. Martínez, J. Phys. Chem. A, 2007, 111, 8500-8598.

55 D. Nachtigallová, A. Aquino, J. Szymczak, M. Barbatti, P. Hobza and H. Lischka, J. Phys. Chem. A, 2011, 115, 52475255.

56 S. Yamazaki and T. Taketsugu, J. Phys. Chem. A, 2012, 116, 491-503.

57 X. Zhang and J. Herbert, J. Phys. Chem. B, 2014, 118, 78067817.

58 S. Faraji, S. Matsika and A. I. Krylov, J. Chem. Phys., 2018, 148, 044103.

59 A. Krylov, J. Phys. Chem. A, 2005, 109, 10638-10645. 